\title{
Diurnal and seasonal changes in IL-6 and IL-18 gene expression in blood leukocytes of male chickens with experimental peritonitis: the impact of lighting conditions and melatonin
}

\author{
E. Turkowskaa ${ }^{1,3}$, S. Rai ${ }^{2,4}$, P.M. Majewski ${ }^{1}$ and K. Skwarło-Sońta ${ }^{1}$ \\ ${ }^{1}$ University of Warsaw, Faculty of Biology, Department of Animal Physiology \\ Miecznikowa 1, 02-096, Warsaw, Poland \\ 2Department of Zoology, School of Life Sciences, Guru Ghasidas Vishwavidyalaya \\ 495009 Bilaspur, Chhattisgarh, India
}

KEY WORDS: melatonin, pineal gland, inflammation, season, interleukins, chickens

Received: 6 November 2012

Revised: 27 February 2013

Accepted: 17 June 2013

${ }^{3}$ Corresponding author:

e-mail: eturkowska@biol.uw.edu.pl

\begin{abstract}
The aim of this study was to examine the involvement of melatonin in the photoperiod-related development of inflammation in chickens. Newly hatched birds obtained in summer and winter were kept in a light : dark (LD) cycle (corresponding to the season of hatch) or in continuous light (LL). Half of the chickens held under $L L$ conditions were given melatonin during the subjective night. Peritonitis was evoked on the $12^{\text {th }}$ day of life by thioglycollate (TG) injection at the beginning or towards the end of the subjective day and the effect was measured $4 \mathrm{~h}$ later, i.e. during the day or at night. The development of inflammation was evaluated by the number of peritoneal leukocytes (PTLs) inflowing to the peritoneal cavity and by the level of mRNAs encoding proinflammatory cytokines in the blood leukocytes. Day/night differences in PTL number were seen only in the summer, regardless of the experimental conditions. In chickens kept under LL conditions and given melatonin, TG injection caused an increase in interleukin-6 (IL-6) mRNA level in blood leukocytes in summer and a decrease in winter. Neither experimental conditions nor circadian time affected the interleukin-18 (IL-18) mRNA level in summer, whereas in winter abundance of this transcript exhibited the daily variations and the influence of the treatment. The obtained results suggest the existence of clockcontrolled diurnal and seasonal variability of the chicken inflammatory reaction, which is influenced in different ways by circulating melatonin.
\end{abstract}

\section{Introduction}

A key function of melatonin (MEL) synthesized rhythmically by the vertebrate pineal gland is to provide information about day/night length and its annual variation. In this way it permits adaptation to predictable environmental changes, like temperature, humidity or food availability (Brandstatter, 2003). As a result, several physiological processes, such as reproduction, fur density and colour, energy expenditure, etc. (Paul et al., 2007) exhibit well-expressed photoperiodic variations throughout the year, and similar seasonality has been recognized in immune function (for a review see Majewski et al., 2012).

${ }^{4}$ Dr. Seema Rai was a Visiting Scientist at the University of Warsaw, Poland, under the INSA-PAS Bilateral Exchange 2012 programme. 
A trade-off between reproduction and immune function has been postulated (Martin et al., 2008) and was confirmed experimentally in seasonal breeders like the jungle bush quail and Indian palm squirrel (Singh and Haldar, 2007; Ahmad and Haldar, 2009). However, the ability of melatonin to synchronize the seasonal immune adaptability of various species is still under investigation. It has been reported that keeping some rodents under different lighting conditions evokes an increase or a decrease in immune tissues weight (i.e. thymus and spleen), demonstrating that melatonin acts directly but differently upon immune function (Nelson and Demas, 1997). Exposure to an extended lighting period or to continuous illumination has been shown to decrease both humoral and cell-mediated immunity in avian species, exemplified by the broiler chicken (Kirby and Froman, 1991) and Japanese quail (Moore and Siopes, 2000). These effects may be due to seasonal changes in the blood melatonin concentration (Kliger et al., 2000), since light intensity itself did not affect immune parameters like B- and T-lymphocyte proliferation, plasma lysozyme concentration or the bacterial killing ability of whole blood (Blatchford et al., 2009).

On the other hand, experimental peritonitis was found to develop differently depending on the season of hatch (winter or summer) in chickens kept under laboratory lighting conditions (LD 12:12) from the day of hatch (Majewski et al., 2005). In particular, the influx of peritoneal leukocytes (PTLs) to the peritoneal cavity was faster and lasted longer in chickens hatched in the summer than in winter birds. These findings, together with decreased levels of reactive oxygen species in PTLs and reduced mitogen-induced splenocyte proliferation, suggest that chicken immunity is season-related and more efficient in summer than in winter (Majewski et al., 2005; Skwarlo-Sonta et al., 2007). This phenomenon does not seem to be limited solely to the chicken, because similar results have been obtained in the Siberian hamster (Phodopus sungorus), a seasonal breeder. In this species, a short day length attenuated the symptoms of infection (Bilbo and Nelson, 2002) and compromised both the development of peritoneal inflammation and mitogen-stimulated splenocyte proliferation (Pawlak et al., 2005, 2009).

It is generally accepted that the diurnal profile of the blood melatonin concentration results from daily variations in the activity of pineal arylalkylamine$\mathrm{N}$-acetyltransferase (AANAT; E.C.2.3.1.87), a key enzyme in melatonin biosynthesis (Klein, 2007). The daily profile of AANAT activity in chickens was found to be affected by the season, regardless of artificial LD 12:12 conditions imposed from the day of hatch (Majewski et al., 2005; Piesiewicz et al., 2012b). This observation suggested the involvement of the endogenous clock, located in the pineal gland of avian species (Bell-Pedersen et al., 2005), and melatonin, in season-related variations in chicken immunity.

Experiments with male chickens have revealed the daily variation in lipopolysaccharide (LPS)-induced systemic inflammation, measured in the spleen by the expression of several cytokines (Naidu et al., 2010). Signaling through cytokines is likely to play a major role in both the activation of innate immunity and the subsequent development of the adaptive response (Commins et al., 2010). The level of mRNAs encoding pro-inflammatory interleukins-6 (IL-6) and -18 (IL-18), known to exert a broad spectrum of biological activities, was evaluated. Chicken IL-6, similarly to its mammalian counterpart, is a multifunctional cytokine produced during systemic inflammation in response to stimuli like LPS, IL-1 $\alpha$ or tumor necrosis factor (TNF- $\alpha$ ) (Akdis et al., 2011). In innate immunity, IL-6 directs leukocyte trafficking and activation, induces the production of acute-phase proteins, and subsequently promotes T-cell proliferation, B-cell differentiation and survival. Unlike most cytokines, IL-18, a member of the IL-1 superfamily, is constitutively expressed and performs an important role in cellular adherence (Commins et al., 2010). The upregulation of several cytokine and chemokine genes, including $I L-6$ and $I L-18$, was recently demonstrated in chicken peripheral blood leukocytes during stress (Shini et al., 2010), suggesting a role for these factors in the stimulation of leukocyte movement and the regulation of inflammatory vs. anti-inflammatory activities.

In the present study, we evaluated the transcription of the $I L-6$ and $I L-18$ genes in peripheral blood leukocytes of chickens hatched in two seasons, in which experimental peritonitis was evoked at the beginning or towards the end of the light phase. It was hypothesized that the photoperiodic conditions corresponding to the season of hatch (LD 16:8 or $8: 16$ in summer and winter, respectively) would modify the development of chicken inflammation in relation to the presence or absence of circulating melatonin. Continuous illumination (LL) was imposed to achieve a functional pinealectomy (John et al., 1994), which was antagonized in a subset of chickens from this LL group by melatonin supplied in the drinking water. We anticipated that photoperiod-related development of inflammation could be modified by the LL conditions, whereas melatonin supplementation should restore the observed season- 
al changes. The development of peritonitis was measured by the number of PTLs infiltrating the peritoneal cavity after injection of an irritant. The level of $I L-6$ and $I L-18$ mRNAs in blood leukocytes was selected as an indicator of systemic inflammation following the recent demonstration of opposite diurnal changes in the expression of these genes in the spleens of LPS-injected chickens (Naidu et al., 2010).

\section{Material and methods}

\section{Animals and experimental design}

Experiments were performed on male Hi-Line chickens hatched in summer (June) and winter (January). On the day of hatch, the chicks were transported from the commercial hatchery (H\&P, Orzesze-Gardawice, Poland) to the animal facility of the Faculty of Biology, University of Warsaw and reared under controlled temperature conditions (32 $\pm 2{ }^{\circ} \mathrm{C}$ for one week and then decreased gradually to $21^{\circ} \mathrm{C}$ ), with free access to the standard feed and water.

Control chickens were reared for 12 days in a LD cycle corresponding to the season of hatch, i.e. LD 16:8 in summer and 8:16 in winter (LD groups). Another group of animals was kept in continuous light (LL group, 250 lux) and half of them received melatonin $\left(15 \mu \mathrm{g} \cdot \mathrm{ml}^{-1}\right.$, Sigma) in their drinking water (LL MEL group), administered for 8 or 16 hours daily in the summer and winter, respectively, to mimic the scotophase. The volume of melatonin solution consumed was recorded daily to calculate the average melatonin dose received by the chickens; this was 1.3 and $2.6 \mathrm{mg} \cdot \mathrm{kg}^{-1} \mathrm{BW} \cdot \cdot$ day $^{-1}$ in summer and winter, respectively.

Experimental peritonitis was initiated in 12-day-old chickens by i.p. injection of a single dose of 3\% sterile thioglycollate (TG group, $2 \mathrm{ml}$. $\left.100 \mathrm{~g}^{-1} \mathrm{BW}\right), 2 \mathrm{~h}$ after the beginning or before the end of the subjective day, as described previously (Piesiewicz et al., 2012a). TG-injected and intact animals (INT group) were decapitated $4 \mathrm{~h}$ later, in the subjective light period (Zeitgeber or Circadian Time, ZT/CT 6) or during darkness (ZT/CT 18 and
10 in summer and winter, respectively). Trunk blood was collected into heparinized tubes for peripheral blood leukocyte isolation. The development of peritonitis was verified by evaluating the number of leukocytes in the peritoneum, obtained by flushing the peritoneal cavity with $7 \mathrm{ml}$ of phosphatebuffered saline (PBS) supplemented with heparin $\left(1 \mu \mathrm{l} \cdot \mathrm{ml}^{-1}\right)$ and counted with a haemocytometer (Majewski et al., 2002, 2005).

All procedures were performed in accordance with the regulations of the Polish Ethical Council for the care and use of laboratory animals and the European Community Directive for the ethical use of experimental animals.

\section{Isolation of peripheral blood leukocytes}

Peripheral blood leukocytes (PBL) were isolated on a Histopaque (Sigma, 1077-1) gradient, according to the manufacturer's protocol. Briefly, blood samples ( $n=4 /$ time point/group) were diluted 1:1 with sterile PBS and an aliquot of $2.5 \mathrm{ml}$ of this mixture was gently loaded onto a $2.5 \mathrm{ml}$ cushion of Histopaque. Following centrifugation (400 g, 30 min, room temperature), the opaque interface containing mononuclear cells was transferred to a clean tube. The cells were washed twice with PBS by centrifugation $(250 \mathrm{~g}, 10 \mathrm{~min})$, then frozen in liquid nitrogen and stored at $-80^{\circ} \mathrm{C}$ prior to further analysis.

\section{Isolation and quantification of mRNA}

Total RNA was isolated from PBLs using an AxyPrep Multisource Total RNA Miniprep Kit and treated with QR1-DNase (Promega). The concentration of RNA was determined spectrophotometrically (NanoDrop-1000, Thermo Scientific) and its quality was assessed by gel electrophoresis. Isolated RNA (40 ng) was then added to one-step RT-qPCR reaction mixtures containing $0.4 \mu \mathrm{l}$ of $50 \times$ Kapa RT Mix (Kapa Sybr Fast Universal qPCR Kit, Kapa Biosystems), $0.5 \mu$ l of gene-specific forward and reverse primers (10 $\mathrm{MM}$, Genomed, Poland; Table 1), $10 \mu$ of $2 \times$ SYBR Green I PCR master mix (Kapa Biosystems) and nuclease free water up to a final volume of $20 \mu \mathrm{l} \cdot$ well $^{-1}$.

Table 1. Sequences of oligonucleotide primers used for qPCR analysis. Primers were designed using OligoPerfect software, based on sequences obtained from the GenBank database

\begin{tabular}{lll}
\hline Gene with GenBank Accession Number & Primer sequence (5' to 3') & Product length \\
\hline Interleukin 6 (IL-6) & F: CAAGGTGACGGAGGAGGAC & 254 bp \\
NM_204628 & R: TGGCGAGGAGGATTTCT & \\
Interleukin 18 (IL-18) & F: GGAATGCGATGCCTTTTG & 264 bp \\
AY_628648 & R: ATTTTCCCATGCTCTTTCTCA & \\
TATA-box binding protein (Tbp) & F: TGTCAGAGCTGCCCCAGATAAG & 163 bp \\
D83135 & R: CAAGTTTGCAACCAAGATTCA & \\
\hline
\end{tabular}


The reactions were set up in 96-well plates and incubated in a Quantica (Techne) thermal cycler, using the following conditions for one-step RT-qPCR: $42^{\circ} \mathrm{C}$ for $10 \mathrm{~min}$ (cDNA synthesis), $95^{\circ} \mathrm{C}$ for $10 \mathrm{~min}$ (reverse transcriptase inactivation), followed by 40 cycles of denaturation $\left(95^{\circ} \mathrm{C}\right.$ for $\left.15 \mathrm{~s}\right)$, annealing $\left(61^{\circ} \mathrm{C}\right.$ for $\left.20 \mathrm{~s}\right)$ and extension $\left(72^{\circ} \mathrm{C}\right.$ for $10 \mathrm{~s}$ ). Each sample was assayed in duplicate. For each RT-qPCR, the dissociation curves were analysed using Quantica software to detect and eliminate possible primer dimers and non-specific amplification. Reactions to prepare standard curves for $I L-6, I L-18$ and the reference gene Tbp (TATAbinding protein) were performed using ten-fold serial dilutions of cDNA fragments that had been separately amplified by PCR. The PCR products were gel purified and the amount was determined spectrophotometrically and verified by sequencing. Cycle threshold $\left(\mathrm{C}_{\mathrm{t}}\right)$ values were used to plot standard curves for Tbp (slope: -3.1866 , y-intercept: 34.585 , $\mathrm{R}^{2}=0.9869$, efficiency: 106\%), IL-6 (slope: -3.0239 , y-intercept: $33.625, \mathrm{R}^{2}=0.9961$, efficiency $114 \%$ ) and IL-18 (slope: -3.4687, y-intercept: 36.84, $\mathrm{R}^{2}=0.9982$, efficiency: 94\%) in which the $\mathrm{C}_{\mathrm{t}}$ values decrease in linear proportion to the log of the template concentration. $\mathrm{C}_{t}$ values from reactions performed with experimental samples were extrapolated from the standard curves in order to determine the initial amount of each particular transcript. These values were then normalized to the level of the Tbp transcript and expressed as the number of target gene mRNA copies per 100 copies of Tbp mRNA. The isolation of RNA and RT-qPCR were performed no later than 1 month after PBL collection.

\section{Statistical analyses}

All data are expressed as the mean \pm SEM. Data were analysed by one-way analysis of variance (ANOVA), followed by the Tukey post-hoc test. The differences were considered significant at $p<0.05$. Statistical analyses were performed using GraphPad Prism 5.02 software.

\section{Results}

\section{PTL number as a measure of peritonitis intensity}

Intraperitoneal injection of TG evoked an influx of PTLs into the peritoneal cavity, which was season- and time of day-dependent, but did not differ between the experimental groups: LD, LL and LL MEL (Figure 1). The elevated PTL number detected in summer (Figure 1A) at ZT/CT 6 ('day') was fol-
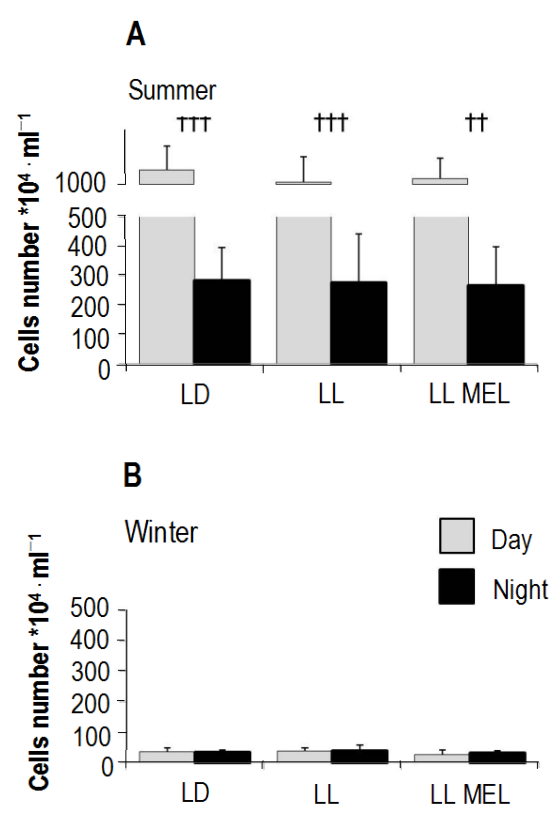

Figure 1. Number of leukocytes isolated from the chicken peritoneal cavity 4 hours after i.p. injection of thioglycollate (TG). Charts show the results obtained for the subjective day (ZT/CT 6, grey bars) and subjective night (ZT/CT 18 and 10 in summer and winter, respectively, black bars). The results are expressed as the mean $\pm S E M, n=10$. Crosses indicate significant differences between day and night in the respective groups (†† $P<0.01$; ††† $P<0.001$ ). ZT/CT - Zeitgeber/ Circadian Time

lowed by a significant decrease at ZT/CT 18 ('night'). In winter, the influx of PTLs was significantly lower $(P<0.001)$, and was the same in all experimental groups and at both time points (Figure 1B).

\section{Influence of peritonitis on the $I L-6$ mRNA level in PBLs}

In summer (Figure 2A), the level of $I L-6$ mRNA measured in the PBLs did not differ between the intact chickens (INT) in the three experimental groups (LD, LL and LL MEL), but was significantly lower at ZT/CT 18 ('night') than at ZT/CT 6 ('day'): $P<0.001$ and $p<0.05$ in the LD and LL MEL groups, respectively. Injection of TG increased the IL-6 mRNA level only in PBLs isolated from chickens that received exogenous melatonin (LL MEL, $P<0.001$ ), having no effect in those from the LD and LL groups, regardless of the time of day (Figure 2A).

In winter (Figure 2B), day/night variations in $I L-6$ transcription in PBLs from intact chickens were seen, with significant differences in the LD $(P<0.001)$ and LL MEL $(p<0.05)$ groups. TG injection caused a significant decrease in PBL IL-6 mRNA in the LL MEL group at CT 10 $(P<0.01)$, and the same tendency was seen in this group at CT 6 and in the LL group at both time points (Figure 2B). 


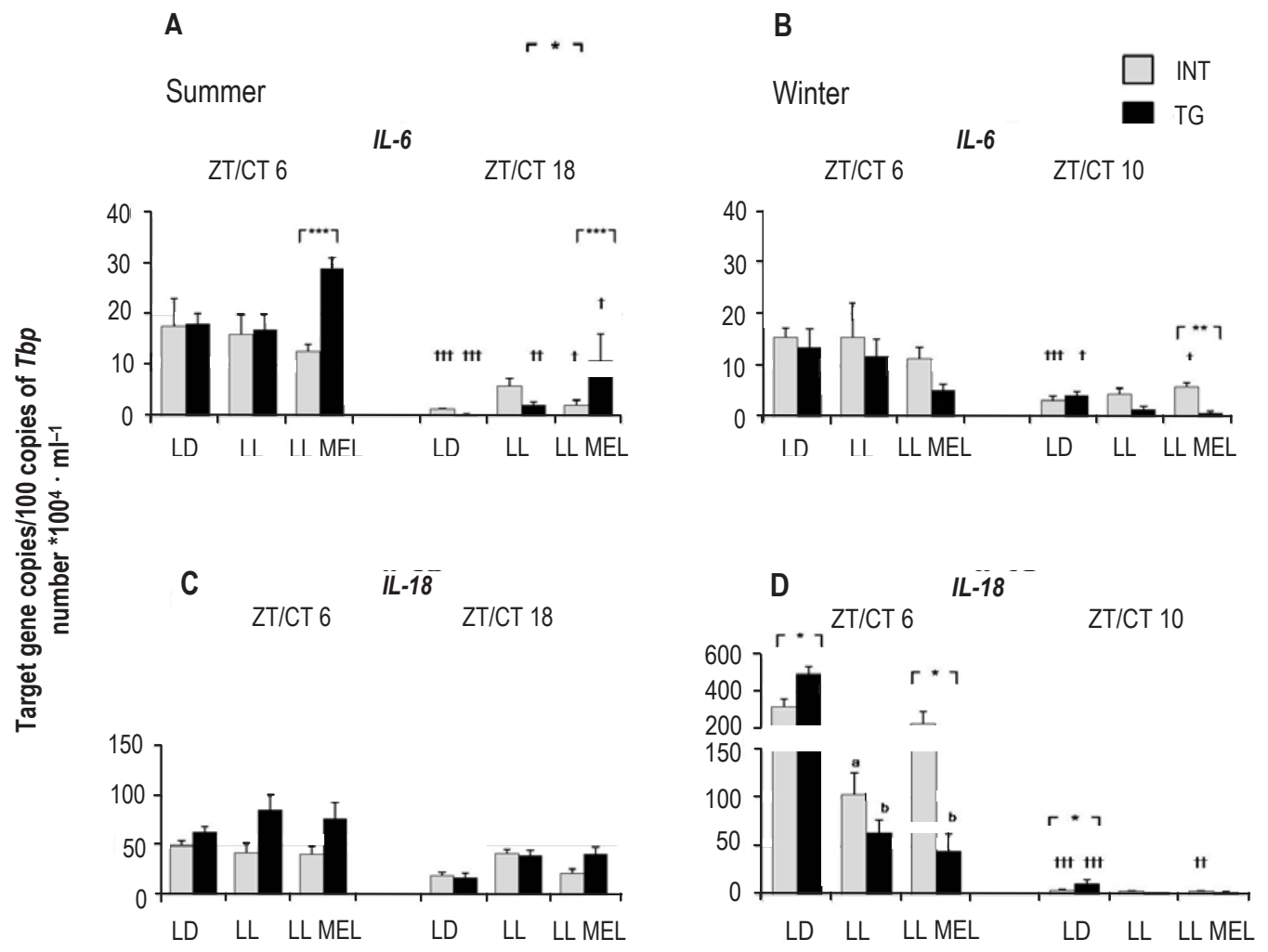

Figure 2. Influence of season (summer vs. winter), circadian time (day vs. night), continuous light (LL), melatonin supplementation (LL MEL) and peritonitis on the level of interleukin-6 (IL-6) and interleukin-18 (IL-18) mRNAs in chicken peritoneal blood leukocytes (PBLs). Peritonitis was elicited by i.p. injection of thioglycollate (TG) at two time points and the transcript levels were evaluated 4 hours later in PBLs from both intact (INT, grey bars) and TG-treated chickens (TG, black bars). The results are expressed as the mean $\pm \mathrm{SEM}, \mathrm{n}=4$ (assayed in duplicate). Asterisks indicate significant differences between INT and TG animals within the same group $\left({ }^{*} p<0.05 ;{ }^{* *} P<0.01 ;{ }^{* * *} P<0.001\right)$, crosses indicate significant differences between day and night within the same season († $p<0.05$; †† $P<0.01$; †† $P<0.001)$. Letter 'a' indicates significant difference between intact animals from light: dark (LD) and LL groups $(P<0.01)$, letter "b" indicates significant difference between TG-injected animals from LD and LL/LL MEL groups $(P<0.001)$. ZT/CT - Zeitgeber/Circadian Time

\section{Influence of peritonitis on the $I L-18$ mRNA level in PBLs}

Neither experimental conditions nor circadian time affected the $I L-18$ mRNA level in PBLs isolated from intact chickens in summer, while in TG-injected birds from all groups at ZT/CT 6 and in the LL MEL group at ZT/CT 18, a tendency towards an increased level of this mRNA was observed (Figure 2C).

A well-pronounced day-night difference in IL-18 mRNA level was observed in winter, with increased transcription during the subjective day, and very low values at subjective night (Figure 2D). In intact chickens at $\mathrm{CT} 6$, LL conditions evoked a decrease in $I L-18$ mRNA level $(P<0.01$, LL vs. LD group) and this effect seems to be reversed in chickens supplemented with melatonin (LL MEL group). After TG-injection the $I L-18$ mRNA level was significantly decreased $(P<0.001)$ under LL conditions, regardless of MEL supplementation. In comparison to the respective INT animals, TGinjection at ZT/CT 6 increased $I L-18$ transcription in the LD group $(p<0.05)$ and decreased it in the LL MEL group $(p<0.05)$, with no change in the LL group.
The levels of $I L-18$ mRNA were very low at ZT/CT 10 , although a significant increase was observed in the LD group after TG injection $(p<0.05)$.

\section{Discussion}

The aim of this study was to examine the season- and time of day-related development of experimental peritonitis in chickens, paying particular attention to the role of melatonin, both endogenous and exogenous. The development of peritonitis was monitored by evaluating both the number of leukocytes infiltrating the site of inflammation and the level of mRNA encoding two pro-inflammatory cytokines (IL-6 and $I L-18$ ) in blood leukocytes. Healthy birds have a very low number of resident leukocytes in their body cavities (Toth and Siegel, 1986), therefore the massive cell influx into the peritoneum in response to the injection of an irritant (TG) is a good measure of the inflammation intensity and its season-related modifications in the chicken (Majewski et al., 2005; Skwarlo-Sonta et al., 2007). On the other hand, although measuring 
cytokine mRNA abundance does not necessarily reflect the real content of bioactive IL- 6 and IL-18 in the blood, a recent report demonstrated that in the absence of an effective avian cytokine bioassay, RT-qPCR is the most sensitive method available to quantify synthesis and regulation of various cytokines (Shini et al., 2010).

As was anticipated using the applied experimental schedule, the influx of inflammatory cells to the peritoneal cavity was strictly season-dependent, i.e. the number of leukocytes isolated $4 \mathrm{~h}$ after TG injection was substantially higher in chickens from the summer experiment compared with those from the winter. This observation corroborates previous findings obtained in different seasons with chickens kept from the day of hatch under controlled LD 12:12 conditions (Majewski et al., 2005). Although conducted using a different lighting schedule, both studies show that in winter it is very hard to stimulate the migration of leukocytes towards the peritoneal cavity. These observations also support the widely accepted concept of seasonality of immunity in various species of mammals and birds (Majewski et al., 2012).

The results of the present study also confirm once more that inflammation evoked during the subjective day is much more intense than that developing at subjective night. Several experimental approaches have been used to show that nocturnal elevation of pineal melatonin biosynthesis is a factor preventing rolling of leukocytes and their access to the site of inflammation, and this anti-inflammatory function of melatonin is now well accepted (e.g. Lotufo et al., 2001; Markus and Ferreira, 2011). Surprisingly, we found that the number of PTLs retrieved at night from the peritoneal cavity of chickens kept in LD conditions (18:6 in summer and 6:18 in winter) did not differ between experimental groups in which the level of melatonin was considered to be quite different: LD vs. LL vs. LL MEL. One possible explanation for this result is that melatonin applied in these experimental conditions is not the main factor controlling immune cell traffic during inflammation, and this aspect will be discussed in detail below. Another interpretation is based on the suggestion that in the experimental schedule employed, the functional pinealectomy was incom-plete in the LL chickens, perhaps because the duration of the constant illumination treatment was too short or melatonin from extra-pineal sources (e.g. retina or Harderian gland) compensated for any deficiency. Experiments to examine the effect of continuous light (LL) on circulating melatonin levels in chickens are currently being performed in our laboratory.
The incomplete pinealectomy scenario seems to be only partially supported by the analysis of levels of mRNAs encoding pro-inflammatory cytokines IL-6 and IL-18 in the PBLs. The expression of both genes was also season- and time of day-dependent, but they responded differently to melatonin presence/absence and TG injection. First, the $I L-18$ gene appeared to be expressed constitutively (at least in the summer experiment), as was found in a previous study (Commins et al., 2010). Second, TG treatment affected the transcription of both cytokine genes only slightly, with the greatest effect observed in the LL MEL group. IL- 6 transcription was enhanced in the summer chickens and decreased in winter birds when stimulation with TG followed 12 days of melatonin treatment, indicating that melatonin can operate as a pro- and an antiinflammatory agent, respectively. A similar, but less clear, seasonal tendency in the $I L-18$ mRNA level was observed in the MEL-supplemented group. It is important to note that the length of the period of melatonin availability in the drinking water corresponded to the seasonal duration of darkness, i.e. to the period of naturally elevated melatonin synthesis ( $8 \mathrm{~h}$ in summer and $16 \mathrm{~h}$ in winter). In the majority of mammalian species examined to date, exogenous melatonin administration in vivo has been found to increase innate immune reactions (Currier et al., 2000) and counteract the immunosuppressive effects of stress (Maestroni, 1999). However, its influence on the development of inflammation appears to be the opposite: in the rodent model, treatment with melatonin decreased the extent of the inflammation (Constantino et al., 1998; Escames et al., 2006). On the other hand, the effect of a short photoperiod (corresponding to the extended melatonin availability in our experiment) on immunity is not clear. Keeping animals under artificial short photoperiod conditions has been shown to enhance selected measures of immunity (Nelson et al., 1995; Ahmad and Haldar, 2009), while field studies have demonstrated that immune system functions are compromised under these conditions (Nelson et al., 1995). These apparently contradictory observations indicate that the regulation of immunity by photoperiod/melatonin is a complex phenomenon that is influenced by the species and varies according to the parameter examined, some being melatonindependent and others not (Wen et al., 2007). Therefore, seasonal variations in immunity cannot be explained by simple changes in circulating melatonin concentration and may be created by the modulation of other factors, for which melatonin is only a timing cue (Majewski et al., 2012). 
This last point raises the role of circadian clock mechanisms in rhythmic immune functions that are now the subject of extensive investigation. Recent studies have shown that in mice, an endogenous clock can influence the daily oscillations in innate immune functions, like bacterial endotoxin-stimulated TNF- $\alpha$ and IL- 6 secretion by isolated spleen cells (Keller et al., 2009) or phagocytosis in macrophages (Hayashi et al., 2007). These circadian clock mechanisms, which can operate autonomously even ex vivo in the spleen and lymph nodes, are based on the expression of major clock genes, like $\mathrm{Bmal}, \mathrm{Per}$, Clock and Rev-erb a (Keller et al., 2009). Moreover, clock gene expression in human immune cells seems to be very sensitive to a damaging challenge, since a single injection with endotoxin was found to suppress the expression of these genes in the PBLs of healthy humans volunteers (Haimovich et al., 2010). Constant light applied to the chickens in the present study desynchronized or shifted the circadian rhythm of the transcription of selected clock genes in the pineal gland, while melatonin supplementation restored the diurnal pattern in a season-dependent manner (data not shown).

Time of day-dependent variations of immune parameters are apparently ubiquitous and have been shown to affect the expression of cytokines in the chicken spleen (Naidu et al., 2010) and in mouse peritoneal macrophages (Hayashi et al., 2007), for example. In the experiments described here, higher levels of both the studied cytokine mRNAs were observed during the subjective daytime vs. nighttime in both seasons and in almost all groups of chickens. This finding is in sharp contrast to the results presented by Naidu et al. (2010), indicating a greater overall induction of both $I L-6$ and $I L-18$ at midnight vs. midday in the chicken spleen during LPS-induced systemic inflammation. Moreover, their study showed that melatonin injected during the day, one hour prior to LPS, evoked a huge increase in pro-inflammatory cytokine gene expression (Naidu et al., 2010). On the contrary, in our experimental model, the administration of melatonin for 12 days before TG treatment caused up-regulation of $I L-6$ expression in summer and down-regulation in winter, and reversed the repressive effect of continuous light on $I L-6$ expression in PBL in summer. However, the experimental protocols employed in these two studies differ in several aspects, including induction of inflammation, melatonin treatment and, most importantly, the source of the immune cells examined. Circulating PBLs represent a highly heterogeneous population of cells that move between the marginal zones and the site of inflammation, and they prob- ably differ in their sensitivity to regulatory/synchronizing humoral factors (Keller et al., 2009).

It has also been suggested that the daily rhythm of the inflammatory response may be attributed either to melatonin or to the anti-inflammatory glucocorticoids (Dickmeis, 2009). Moreover, the compromised immune status observed in Siberian hamsters under short day conditions (LD 8:16) was shown to result from the combined action of elevated melatonin, high cortisol and low testosterone concentrations in the serum (Pawlak et al., 2009). Considering the available data, it may be concluded that diurnal and seasonal changes in immunity are related to the general neuro-endocrine status of the organism (Haldar et al., 2008).

To summarize, the results of the present study suggest that the seasonal and daily changes in the development of acute inflammation can be driven by local autonomous clock machinery, uncoupled from the activity of the central pineal clock and the level of melatonin in the circulation. A greater understanding of the bi-directional communication between the immune system and the circadian clock will provide valuable insight into the timing mechanism of physiological and pathophysiological immune functions.

\section{Conclusions}

Using the described experimental schedule, the greatest TG-evoked peritonitis was observed in chickens in the summer and during the subjective day, although these variations were not closely correlated with melatonin treatment. The transcription of genes encoding the proinflammatory cytokines IL-6 and IL- 18 in peripheral blood leucocytes (PBLs) was only slightly modified by inflammation, with more pronounced daily and seasonal changes. However, melatonin can function as both a pro- and anti-inflammatory agent, and its action may change throughout the year. Our results, together with recently published data, suggest that the development of inflammation at least in chicken could be driven more by the clock system than by melatonin itself.

\section{Acknowledgment}

This work was performed within the framework of Polish Ministry of Science and Higher Education Grant No.NN303 503438 and Faculty of Biology Grant BW 501/86-102355.

Results were presented on the Workshop 'Central and local mechanisms of growth and development', Jablonna 18-19 October 2012. 


\section{References}

Ahmad R., Haldar C., 2009. Photoperiod-testicular-immune interaction in a seasonal breeder Indian palm squirrel Funambulus pennanti during the reproductively inactive and active phases. J. Neuroendocrinol. 21, 2-9

Akdis M., Burgler S., Crameri R., et al. 2011. Interleukins, from 1 to 37 , and interferon- $\gamma$ : receptors, functions, and roles in diseases. J. Allerg. Clin. Immunol. 127, 701-721.e1-70

Bell-Pedersen D., Cassone V.M., Earnest D.J., Golden S.S., Hardin P.E., Thomas T.L., Zoran M.J., 2005. Circadian rhythms from multiple oscillators: lessons from diverse organisms. Nat. Rev. Genet. 6, 544-556

Bilbo S.D., Nelson R.J., 2002. Melatonin regulates energy balance and attenuates fever in Siberian hamsters. Endocrinology $143,2527-2533$

Blatchford R.A., Klasing K.C., Shivaprasad H.L., Wakenell P.S., Archer G.S., Mench J.A., 2009. The effect of light intensity on the behavior, eye and leg health, and immune function of broiler chickens. Poultry Sci. 88, 20-28

Brandstätter R., 2003. Encoding time of day and time of year by the avian circadian system. J. Neuroendocrinol. 15, 398-404

Commins S.P., Borish L., Steinke J.W., 2010. Immunologic messenger molecules: cytokines, interferons, and chemokines. J. Allerg. Clin. Immunol. 125 Suppl. 2, S53-S72

Constantino G., Cuzzocrea S., Mazzon E., Caputi A.P., 1998. Protective effects of melatonin in zymosan-activated plasmainduced paw inflammation. Eur. J. Pharmacol. 363, 57-63

Currier N.L., Sun L.Z., Miller S.C., 2000. Exogenous melatonin: Quantitative enhancement in vivo of cells mediating nonspecific immunity. J. Neuroimmunol. 104, 101-108

Dickmeis T., 2009. Glucocorticoids and the circadian clock. J. Endocrinol. 200, 3-22

Escames G., Acuña-Castroviejo D., López L.C., Tan D.X., Maldonado M.D., Sánchez-Hidalgo M., León J., Reiter R.J., 2006. Pharmacological utility of melatonin in the treatment of septic shock: Experimental and clinical evidence. J. Pharm. Pharmacol. 58, 1153-1165

Haimovich B., Calvano J., Haimovich A.D., Calvano S.E., Coyle S.M., Lowry S.F., 2010. In vivo endotoxin synchronizes and suppresses clock gene expression in human peripheral blood leukocytes. Crit. Care Med. 38, 751-758

Haldar C., Singh S., Rai S., Skwarlo-Sonta K., Pawlak J., Singaravel M., 2008. Melatonin an immunomodulation: Involvement of the neuro-endocrine network. In: C. Haldar, M. Singaravel, S.R. Pandi-Perumal, D.P. Cardinali (Editors). Experimental Endocrinology and Reproductive Biology. Science Publishers, Plymouth (UK), pp. 297-314

Hayashi M., Shimba S., Tezuka M., 2007. Characterization of the molecular clock in mouse peritoneal macrophages. Biol. Pharm. Bull. 30, 621-626

John T.M., Brown M.C., Wideman L., Brown G.M., 1994. Melatonin replacement nullifies the effect of light-induced functional pinealectomy on nociceptive rhythm in the rat. Physiol. Behav. 55, 735-739

Keller M., Mazuch J., Abraham U., Eom G.D., Herzog E.D., Volk H.D., Kramer A., Maier B., 2009. A circadian clock in macrophages controls inflammatory immune responses. Proc. Nat. Acad. Sci. USA 106, 21407-21412

Kirby J.D., Froman D.P., 1991. Research note: evaluation of humoral and delayed hypersensitivity responses in cockerels reared under constant light or a twelve hour light:twelve hour dark photoperiod. Poultry Sci. 70, 2375-2378
Klein D.C., 2007. Arylalkylamine N-Acetyltransferase: 'the Timezyme'. J. Biol. Chem. 282, 4233-4237

Kliger C.A., Gehad A.E., Hulet R.M., Roush W.B., Lillehoj H.S., Mashaly M.M., 2000. Effects of photoperiod and melatonin on lymphocyte activities in male broiler chickens. Poultry Sci. $79,18-25$

Lotufo C.M., Lopes C., Dubocovich M.L., Farsky S.H., Markus R.P., 2001. Melatonin and $\mathrm{N}$-acetylserotonin inhibit leukocyte rolling and adhesion to rat microcirculation. Eur. J. Pharmacol. 430, 351-357

Maestroni G.J., 1999. MLT and the immune-hematopoietic system. Adv. Exp. Med. Biol. 460, 395-405

Majewski P., Adamska I., Pawlak J., Baranska A., Skwarlo-Sonta K., 2005. Seasonality of pineal gland activity and immune functions in chickens. J. Pineal Res. 39, 66-72

Majewski P., Markowska M., Laskowska H., Waloch M., SkwarloSonta K., 2002. Effect of morphine on thioglycollate-induced peritonitis in chickens, Neuroendocrinol. Lett. 23,161-167

Majewski P., Markowska M., Pawlak J., Piesiewicz A., Turkowska E., Skwarlo-Sonta K., 2012. Pineal gland and melatonin: Impact on the seasonality of immune defence in mammals and birds. Adv. Neuroimmunol. Biol. 3, 95-108

Markus R.P., Ferreira Z.S., 2011. The immune-pineal axis: the role of pineal and extra-pineal melatonin in modulating inflammation. Adv. Neuroimmunol. Biol. 1, 95-104

Martin L.B., Weil Z.M., Nelson R.J., 2008. Seasonal changes in vertebrate immune activity: Mediation by physiological tradeoffs. Phil. Trans. Roy. Soc. London B. 363 (1490), 321-339

Moore C.B., Siopes T.D., 2000. Effects of lighting conditions and melatonin supplementation on the cellular and humoral immune responses in Japanese quail Coturnix coturnix japonica. Gen. Comp. Endocrinol. 119, 95-104

Naidu K.S., Morgan L.W., Bailey M.J., 2010. Inflammation in the avian spleen: timing is everything. BMC Mol. Biol. 11, 104-116

Nelson R.J., Demas G.E., 1997. Role of melatonin in mediating seasonal energetic and immunologic adaptations. Brain Res. Bull. 44, 423-430

Nelson R.J., Demas G.E., Klein S.L., Kriegsfeld L.J., 1995. The influence of season, photoperiod, and pineal melatonin on immune function. J. Pineal Res. 19, 149-165

Paul M.J., George N.T., Zucker I., Butler M.P., 2007. Photoperiodic and hormonal influences on fur density and regrowth in two hamster species. Amer. J. Physiol. - Regul. Integr. C 293, R2363-2369

Pawlak J., Golab M., Markowska M., Majewski P., Skwarlo-Sonta K., 2009. Photoperiod-related changes in hormonal and immune status of male Siberian hamsters, Phodopus sungorus. Comp. Biochem. Physiol. PT A 152, 299-303

Pawlak J., Majewski P., Markowska M., Skwarlo-Sonta K., 2005. Season- and gender-dependent changes in the immune function of Siberian hamsters (Phodopus sungorus). Neuroendocrinol. Lett. 26, 55-60

Piesiewicz A., Kedzierska U., Adamska I., Usarek M., Zeman M., Skwarlo-Sonta K., Majewski P.M., 2012a. Pineal arylalkylamine $\mathrm{N}$-acetyltransferase (Aanat) gene expression as a target of inflammatory mediators in the chicken. Gen. Comp. Endocrinol. 179, 143-151

Piesiewicz A., Kedzierska U., Podobas E., Adamska I., Zuzewicz K., Majewski P.M., 2012b. Season-dependent postembryonic maturation of the diurnal rhythm of melatonin biosynthesis in the chicken pineal gland. Chronobiol. Int. 29, 1227-1238

Shini S., Shini A., Kaiser P., 2010. Cytokine and chemokine gene expression profiles in heterophils from chickens treated with corticosterone. Stress 13, 185-194 
Singh S.S., Haldar C., 2007. Peripheral melatonin modulates seasonal immunity and reproduction of Indian tropical male bird Perdicula asiatica. Comp. Biochem. Physiol. PT A. 146, 446-450

Skwarlo-Sonta K., Majewski P., Pawlak J., Markowska M., 2007. Photoperiodic vs. non-photoperiodic animals - circadian and seasonal changes in immunity. In: S.R. Pandi-Perumal, D.P. Cardinali (Editors). Melatonin: From molecules to Therapy. New York, Nova Science Publishers Inc., pp. 247-260
Toth T.E., Siegel P.B., 1986. Cellular defense of the avian respiratory tract: paucity of free-residing macrophages in the normal chicken. Avian Dis. 30, 67-75

Wen J.C., Dhabhar F.S., Prendergast B.J., 2007. Pineal-dependent and -independent effects of photoperiod on immune function in Siberian hamsters (Phodopus sungorus). Hormone. Behav. $51,31-39$ 